\title{
Precise Measurement of RDCs in Water and DMSO Based Gels Using a Silicone Rubber Tube for Tunable Stretching
}

\author{
Grit Kummerlöwe, Felix Halbach, Burkhardt Laufer and Burkhard Luy*
}

\author{
Technische Universität München, Department Chemie, Lehrstuhl II für Organische Chemie, Garching, Germany
}

\begin{abstract}
Residual dipolar couplings (RDCs) are of great interest for the structure determination of biomacromolecules as well as of organic molecules like synthetic or natural products. Their accurate measurement requires a proper degree of alignment for the molecule under investigation. As has been shown recently, a stretching device based on a flexible silicone rubber tube provides an easy, rapid, and reversible variation of alignment strength. We show in this article that such an apparatus is not limited to gelatin, but can also be used with covalently cross-linked hydrogels and even gels with polar organic solvents like DMSO. Using sucrose and a cyclic hexapeptide, we were able to demonstrate that the approximately linear relation of alignment with the extension factor of the stretched gel allows the measurement of RDCs with high precision if corresponding spectra are acquired at various alignment strengths. The method seems to be widely applicable to the structure determination of molecules of arbitrary size using standard $5 \mathrm{~mm}$ NMR equipment.
\end{abstract}

\section{INTRODUCTION}

Residual dipolar couplings (RDCs) with potentially high structural information content can be measured in partially aligned samples for which so-called alignment media are needed [1-3]. Next to classical liquid crystalline phases, mechanically stretched polymer gels form a second class of alignment media [4] which are applicable to a wide range of solutes and solvents [5-12]. Based on gelatin as the polymer gel [13], Kuchel et al. recently developed a remarkable apparatus for rapid and reversible gel stretching [14] and demonstrated its usefulness by the distinction of enantiomers using RDCs and other anisotropic parameters [15].

Here, we will demonstrate that the stretching device is not limited to gelatin but can also be used with other aqueous gels and even DMSO-based gels. Furthermore, using sucrose and a cyclic hexapeptide as easily accessible test samples, we will show that the scalability of anisotropic parameters with respect to the extension factor allows the precise measurement of RDCs.

\section{STRETCHING APPARATUS AND GEL PREPARA- TION}

Following the fundamental principles of Kuchel et al. [14], we redesigned a stretching apparatus to fit into a standard $5 \mathrm{~mm}$ high resolution probehead as shown in Fig. (1). Silicone tubing (high quality tubing, VWR, outer diameter 4 $\mathrm{mm}$, inner diameter $2.4 \mathrm{~mm}$ ), placed inside an open-cut NMR-tube and fixed with a PTFE plug at the bottom, is used as an expandable container for the swollen gel with the solute molecule inside. When stretching the silicone tubing, the polymer gel is stretched as well, which in turn leads to partial alignment and measurable anisotropic NMR-parameters.

*Address correspondence to these authors at the Technische Universität München, Department Chemie, Lehrstuhl II für Organische Chemie, Lichtenbergstr. 4, D-85747, Garching, Germany;

E-mail: Burkhard.Luy@ch.tum.de
For verification of the alignment properties of the stretching apparatus, we prepared a $40 \%(\mathrm{w} / \mathrm{v})$ sample of porcine gelatin with an approximate Bloomgrade of 250 dissolved at $50^{\circ} \mathrm{C}$ in $\mathrm{D}_{2} \mathrm{O}$. As a solute molecule for measuring RDCs we added $43 \mathrm{mg}$ of sucrose to a final concentration of approximately $500 \mathrm{mM}$. Gelatin is a special gel which can be recasted inside the rubber tube by a simple heating and recooling cycle, as it is cross-linked via hydrogen bonds only. In order to test how far the apparatus is applicable to conventional polymer gels with covalent cross-linking, we prepared several gel samples using published protocols with gel diameters adjusted to accomodate for the dimensions of the stretching apparatus. The results presented in this article were obtained using the following gel preparations:

The poly(acrylamide) (PAA) gel was prepared from a solution of $8.55 \%(\mathrm{w} / \mathrm{v})$ acrylamide and $0.45 \%(\mathrm{w} / \mathrm{v}) \mathrm{N}, \mathrm{N}^{6}$ methylenebis(acrylamide) with $0.1 \%(\mathrm{w} / \mathrm{v})$ ammonium persulfate and $0.1 \%(\mathrm{v} / \mathrm{v}) \mathrm{N}, \mathrm{N}, \mathrm{N}^{\circ}, \mathrm{N}^{\circ}$-tetramethylethylendiamin for cross-linking [5]. Radical polymerization was allowed for $1 \mathrm{~h}$ within glass tubes of $2.4 \mathrm{~mm}$ inner diameter. Gels were washed three times with water to remove free acrylamide before they were dried at room temperature.

Poly(acrylonitrile) (PAN) gels were obtained by crosslinking PAN with accelerated electrons [11]. For preparation a PAN/DMSO- $\mathrm{d}_{6}$-solution $(280 \mathrm{~g} / \mathrm{L})$ was filled in PTFE tubes of $2.4 \mathrm{~mm}$ inner diameter and irradiated with $10 \mathrm{MeV}$ electrons. Total irradiation doses were $320 \mathrm{kGy}$ (for the sucrose sample) and $480 \mathrm{kGy}$ (for the peptide sample).

Dried polymer sticks were placed inside the silicone tubing and a solution $(\sim 100 \mu \mathrm{L})$ of the substance of interest (500 $\mathrm{mM}$ sucrose in $\mathrm{D}_{2} \mathrm{O}$ for PAA gel, $200 \mathrm{mM}$ sucrose in DMSO- $\mathrm{d}_{6}$ and $50 \mathrm{mM}$ peptide in $\mathrm{DMSO}-\mathrm{d}_{6}$ for the PAN gels) was added. Samples were allowed to swell and equilibrate for approximately 48 hours.

All prepared samples could easily be stretched by the silicone-based stretching device with very good long-term stability of the hydro gels. The PAN/DMSO samples, in our 
experience, were stable for more than two weeks and coupling measurements could easily be achieved. However, after about one month the gels suddenly started to shrink to their original, dry size. We looked at this effect in more detail: six similar PAN/DMSO gels were placed in small glass vials which were evacuated and flushed with argon several times to ensure an inert atmosphere. Subsequently, half of the gels were covered with dry DMSO to see if residual DMSO on top of the gel has an influence. For each of the equivalent sample preparations, one of three gels was kept under argon, one was given access to air by opening the vial and to one water was added. Gels stored under argon were stable over several months. The gel with free access to air shrank within a few days, the one only covered by DMSO within a few weeks. When water was added to the gels, the effect was the strongest: the one not covered by DMSO shrank instantaneously, while the one covered with DMSO started to shrink just after a critical concentration of water was reached and within few hours had returned to its dry size. The experiments lead us to the interpretation that the stretching device allows water in the form of humidity to diffuse slowly via the silicone rubber tubing into the gel which then is responsible for the observed gel shrinking.

\section{RDC MEASUREMENT}

After preparing the polymer gel samples in the stretching apparatus, we decided to measure heteronuclear ${ }^{1} \mathrm{H},{ }^{13} \mathrm{C}$ onebond couplings that contain the most easily accessible RDCs. For their measurement we used the so-called CLIPHSQC pulse sequence, a $t_{2}$-coupled HSQC with pure absorption lineshapes [16]. Experiments for sucrose dissolved in the various gels were acquired on a Bruker DMX $600 \mathrm{MHz}$ spectrometer, using the hard pulse version of the CLIPHSQC as described in [16]. $8192 \times 128$ data points were recorded with spectral widths of $8 \mathrm{ppm}\left({ }^{1} \mathrm{H}\right)$ and $50 \mathrm{ppm}$ $\left({ }^{13} \mathrm{C}\right)$, respectively.

Experiments concerning the peptide cyclo(-Arg-NalAla-Gly-D-Tyr-Arg-) (Nal = 2-Naphthylalanine) were implemented on a Bruker Avance $500 \mathrm{MHz}$ spectrometer with
$4096 \times 512$ data points in the ${ }^{1} \mathrm{H}$ and ${ }^{13} \mathrm{C}$ dimension and corresponding spectral widths of 10 and $80 \mathrm{ppm}$. The pulse sequence was implemented using broadband excitation and inversion pulses as described in [16] (BEBOP and BIBOP pulses of 337.5 and $307.5 \mu \mathrm{s}$ and $10 \mathrm{kHz}$ rf-amplitude [17]) on the carbon channel, allowing the center of the carbon spectrum to be set at $40 \mathrm{ppm}$ with aromatic signals folded into the spectrum without loss in sensitivity. Depending on the degree of stretching, 72-104 scans per increment were recorded, leading to an overall experiment duration of 14$20 \mathrm{~h}$ per experiment.

The resulting CLIP-HSQC of sucrose in unstretched gelatin at $293 \mathrm{~K}$ is shown in Fig. (2) as an example. While the methylene moieties show identical proton chemical shifts and do not allow reliable coupling extraction, all methine heteronuclear one-bond couplings can be measured from non-overlapping signals. For two signals corresponding to $\mathrm{H} 1$ ' $-\mathrm{Cl}$ ' of the glucose ring and $\mathrm{H} 5-\mathrm{C} 5$ of the fructose ring, the cross peaks are also shown in the insets at various stages of stretching of the silicone tubing. Cross peaks drawn in blue indicate stretched gelatin, while red cross peaks mark spectra measured in compressed gelatin. Gel compression in this case was achieved by heating the fully stretched gelatin sample to $50^{\circ} \mathrm{C}$ and subsequently releasing the recooled silicone tubing. As expected, the compressed gel shows anisotropic parameters with opposite sign compared to the stretched gel.

The extracted one-bond heteronuclear couplings ${ }^{1} \mathrm{~T}_{\mathrm{CH}}=$ ${ }^{1} \mathrm{~J}_{\mathrm{CH}}+\mathrm{D}_{\mathrm{CH}}$ are shown in Fig. (3A). Clearly, the approximately linear relation of the measured $\mathrm{D}_{\mathrm{CH}}$ couplings with respect to the extension factor $\Xi$ (the difference in length between the stretched and unstretched gel divided by the length of the unstretched gel) [15], and the quadrupolar splitting of the solvent $\Delta v_{\mathrm{Q}}$ are evident. This finding corroborates the original data on the measurement of ${ }^{23} \mathrm{Na}^{+}$and ${ }^{133} \mathrm{Cs}^{+}$ quadrupolar coupling constants using the stretching apparatus [14]. The about linear scalability appears to be a general property of the stretching device.

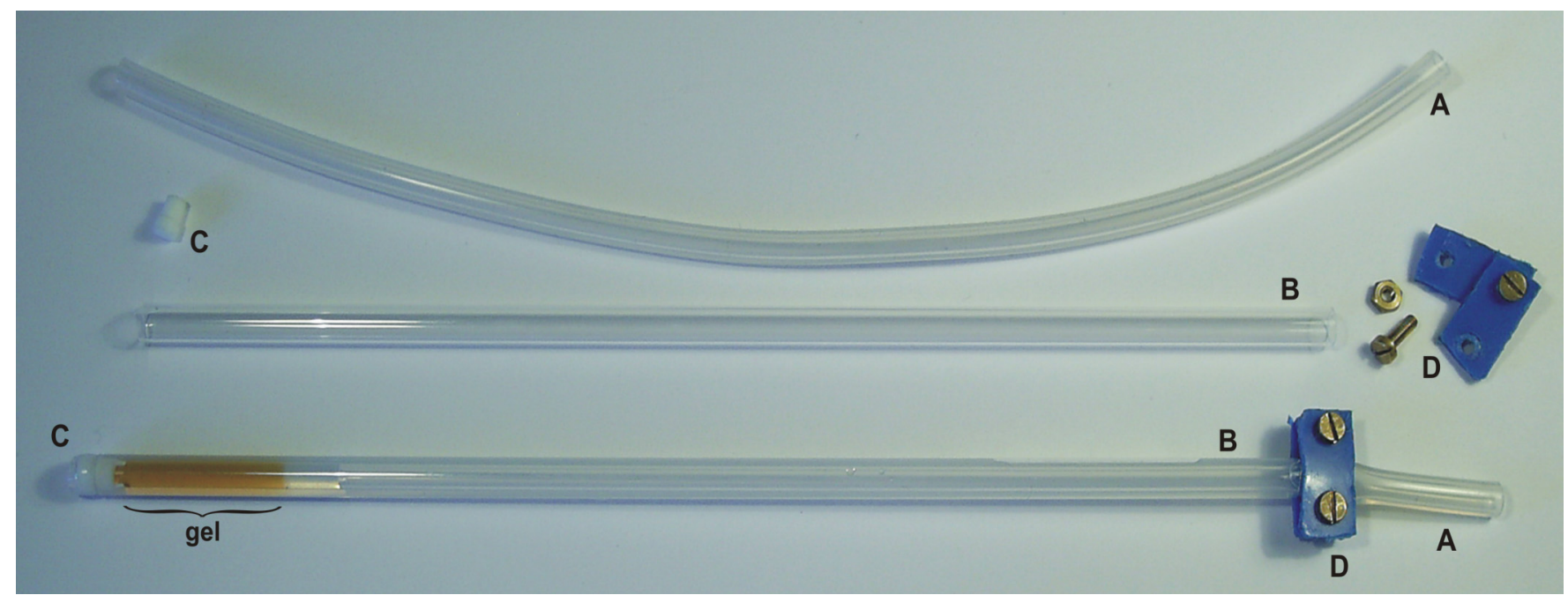

Fig. (1). Stretching apparatus redesigned for $5 \mathrm{~mm}$ standard high resolution NMR probe heads. The flexible silicone tube (A) is placed inside an open-cut $5 \mathrm{~mm}$ NMR tube (B) and fixed with a PTFE plug (C) at the bottom. Plastic clamp and brass screws (D) are used to fixate the stretched silicone tube at the top of the device. Inside the assembled apparatus a reddish-brown poly(acrylonitrile)/DMSO gel is ready to be stretched. The stretching of the gel is demonstrated in the supporting information. 


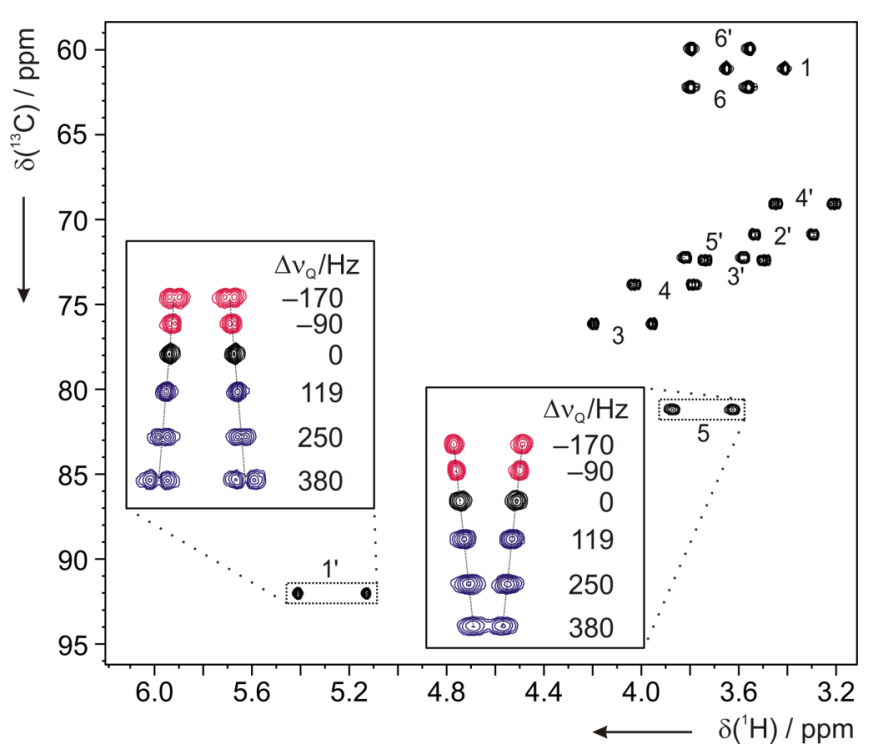

Fig. (2). CLIP-HSQC [16] of sucrose in $40 \%$ gelatin. The black spectrum corresponds to the isotropic stage (non-stretched). Peaks 1 to 6 belong to the fructose ring, while 1' to 6' belong to the glucose ring of sucrose as named within the structure shown in Fig. (3). Insets show two signals (C1'-H1' and C5-H5) of various stages of stretching (blue) and compression (red). Peaks are separated in the vertical direction according to the corresponding quadrupolar splitting $\Delta v_{\mathrm{Q}}$ of the solvent $\mathrm{D}_{2} \mathrm{O}$. The linear dependence between observed ${ }^{1} \mathrm{~T}_{\mathrm{CH}}$-couplings and the quadrupolar splittings is evident.

The ${ }^{1} \mathrm{~T}_{\mathrm{CH}}$ coupling constants were extracted by selecting a slice of the CLIP-HSQC at the appropriate carbon frequency and manually shifting a copy of the slice until the corresponding multiplet components were centered with respect to each other. Although the signal to noise ratio of the sucrose spectra seems to be sufficient for a highly accurate coupling measurement, extracted couplings deviate up to 2.6 $\mathrm{Hz}$ from the linear fit. For most deviations, the choice of ${ }^{13} \mathrm{C}$ frequency for the slice and the variation of line shapes within the multiplets (due to spectral artifacts or slight second order contributions [16]) seem to be responsible for the differences in individual coupling extractions. However, due to the linear relationship of RDCs with the quadrupolar splitting of the deuterated solvent, a linear fit through couplings measured at different stretchings leads to a slope that can be extracted with a significantly higher precision than would be possible from individual experiments. This slope is directly proportional to the RDCs and represents a parameter that is independent of a specific alignment strength.

RDC measurement using the stretching apparatus is not limited to gelatin as the orienting medium. Covalently crosslinked poly(acrylamide) prepared e.g. as described in the previous section is well-known as an alignment medium for water-soluble organic molecules and biomacromolecules [5, $6,18,19]$, and, if the diameter of the precast gel is adjusted to fit inside the silicone tube, can also be used in the rubberbased gel stretching apparatus. RDC measurements on a $9 \%$ poly(acrylamide) sample containing approximately $500 \mathrm{mM}$ sucrose were carried out in the same way as described for gelatin. Since the hydrogel is covalently cross-linked, heating up the gel will not change its shape and only measurements of the stretched gel are possible. The resulting ${ }^{1} \mathrm{~T}_{\mathrm{CH}}$ coupling constants for sucrose are shown in Fig. (3B) with respect to $\Delta v_{\mathrm{Q}}$. Interestingly, the slopes of individual methine groups are different for PAA as compared to gelatin, indicating different resulting alignment tensors for the two media.

DMSO is an important solvent for pharmaceutical and many synthetic applications and we were interested to test the compatibility of the stretching device with this polar organic solvent. Silicone rubber tubing does not swell or change its shape in any way when it is brought in contact with DMSO and indeed RDC measurements using poly(acrylonitrile)/DMSO- $\mathrm{d}_{6}$ gels [11] are possible. With the gel prepared as described in the previous section and using the same measurement procedures as for gelatin and PAA, ${ }^{1} \mathrm{~T}_{\mathrm{CH}}$ coupling constants for sucrose could be extracted in PAN/DMSO- $\mathrm{d}_{6}$ as shown in Fig. (3C).

As a second test molecule we used the small cyclic peptide $\operatorname{cyclo}(-\mathrm{Arg}-\mathrm{Nal}-\mathrm{Ala}-\mathrm{Gly}-D$-Tyr-Arg-) (Nal = 2-Naphthylalanine). The peptide is soluble in DMSO and we prepared a sample with a PAN/DMSO- $\mathrm{d}_{6}$ gel as described above. Like for sucrose we acquired several CLIP-HSQC spectra at different stages of stretching and measured corresponding ${ }^{1} \mathrm{~T}_{\mathrm{CH}}$ coupling constants for all methine and methyl groups (Fig. 4).

\section{DISCUSSION}

We have demonstrated that the stretching apparatus originally developed by Kuchel et al. [14] can be used to precisely measure residual dipolar couplings due to the scalability of the anisotropic parameters with the extension factor $\Xi$. As shown for sucrose, the linear relationship can be used to fit the slope with very high precision from a number of CLIP-HSQC experiments. RDCs and in particular the scaling of alignment might also be used to vary overlap among signals of interest [20], which gives another tool to enhance the accuracy of the desired dipolar couplings.

The downside of the stretching apparatus designed for 5 mm NMR-tubes is, of course, decreased sample volume. Since the silicone rubber tube is placed inside the conventional glass tube, the active sample volume is considerably reduced. This is even more the case when the rubber tube is stretched in order to be able to measure RDCs. Compared to a conventional gel sample made of a polymer stick swollen inside a standard $5 \mathrm{~mm}$ tube [21], the sensitivity decreases approximately by a factor of 3 to 5 , depending on the degree of stretching. Specially designed silicone tubings with thinner walls and larger diameters might improve the situation.

When setting up the apparatus, one has to take care in choosing the right silicone rubber tubing. In our experience some silicone tubings have non-concentric holes and very rough surfaces which significantly reduce shimming capabilities. We also found tubing with paramagnetic filling material that resulted in spectra with line widths of $40 \mathrm{~Hz}$ and broader. With the high quality silicone tubing used in the experiments shown here, we were able to generally obtain linewidths of about $2 \mathrm{~Hz}$ which improved even further for higher extension factors, as the active volume decreases and the sample in the center of the $\mathrm{B}_{0}$-field is more easily shimmed. Of course, multiplet complexity might considerably increase upon alignment (see e.g. linewidth considerations in [7]), in which case the broad effective signal widths cannot be attributed to the stretching apparatus. 

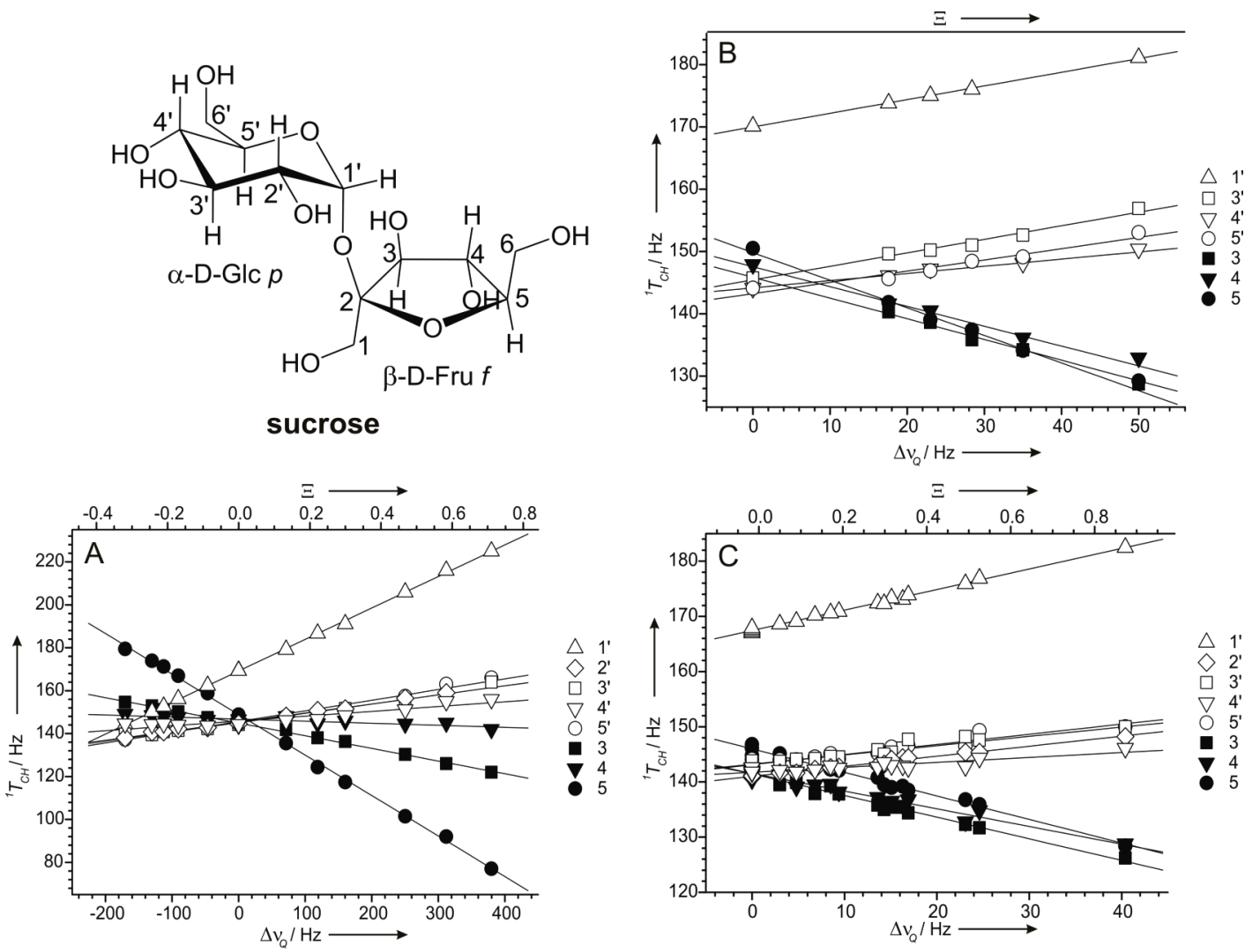

Fig. (3). The dependence of one-bond CH-couplings $\left({ }^{1} \mathrm{~T}_{\mathrm{CH}}={ }^{1} \mathrm{~J}_{\mathrm{CH}}+\mathrm{D}_{\mathrm{CH}}\right)$ of sucrose on the quadrupolar splitting $\left(\Delta v_{\mathrm{Q}}\right)$ of the solvent and the extension factor $(\Xi)$ of the used gels. The slopes of individual $\mathrm{CH}$-groups differ for sucrose in gelatin/ $\mathrm{D}_{2} \mathrm{O}(\mathbf{A})$, compared to sucrose in $\mathrm{PAA} / \mathrm{D}_{2} \mathrm{O}(\mathbf{B})$ and in PAN/DMSO $(\mathbf{C})$ indicating different alignment or conformational properties of the solute.

The stretching device with its rapid and reversible scaling of alignment significantly improves the flexibility and accuracy of RDC measurements. Long-range ${ }^{1} \mathrm{H},{ }^{13} \mathrm{C}$ and onebond ${ }^{13} \mathrm{C},{ }^{13} \mathrm{C}$ RDCs, for example, are approximately one order of magnitude smaller than corresponding heteronuclear ${ }^{1} \mathrm{H},{ }^{13} \mathrm{C}$ one-bond couplings. With the stretching apparatus it should be possible to tune the size of RDCs in such a way that optimal conditions for their measurement are obtained. In contrast to other procedures, like the scaling using variable angle sample spinning [22, 23], standard liquid state NMR equipment with its superior $\mathrm{B}_{0}$-homogeneity can be used.

The method is not limited to sucrose and the peptide shown, but by now has been applied to several other samples in our laboratory including natural products and uniformly isotope-labeled proteins (data not shown). The stretching apparatus seems to be applicable whenever the solute molecule diffuses into a specific gel and this gel does not influence the stretching capability of the rubber tubing. Silicone rubber tubing appears to be compatible with practically all aqueous gels and also allows measurements using highly polar organic solvent-based gels like PAN/DMSO. However, silicone tubing, especially in the stretched state, seems to allow water to diffuse inside the sample. This implies limitations when a sample has to be free of water. Since silicone rubber swells in most apolar organic solvents (see e.g. [8, 24], where silicone is used for gel preparation), it cannot be used inside the stretching apparatus for solvents like chloroform, benzene, or dichloromethane. Tubing made out of fluorinated elastomer materials as e.g. Viton ${ }^{\circledR}$ or Kalrez ${ }^{\circledR}$ might solve this solvent compatibility issue in the future.

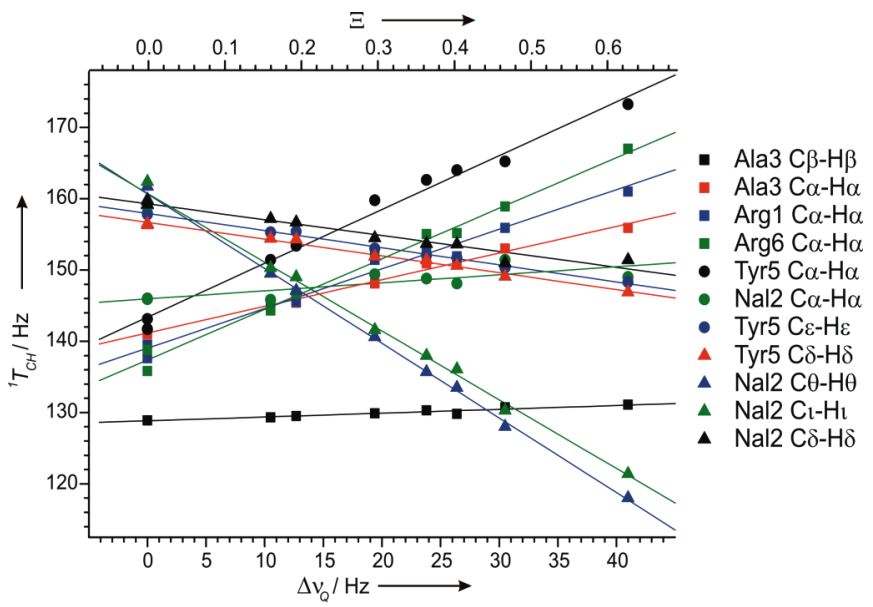

Fig. (4). One-bond ${ }^{1} \mathrm{~T}_{\mathrm{CH}}$-couplings of methine and methyl groups of cyclo(-Arg-Nal-Ala-Gly-D-Tyr-Arg-) with respect to the quadrupolar splitting $\left(\Delta v_{\mathrm{Q}}\right)$ of DMSO- $\mathrm{d}_{6}$ (bottom axis) and the extension factor ( $\Xi$ ) of the PAN gel (top axis). For the assignment of the peptide see the supporting information.

Any kind of alignment medium, if based on liquid crystalline phases or stretched polymer gels, might influence the average conformation of a given solute molecule. When measuring couplings in isotropic solution and inside the alignment medium, conformational changes in the two different environments can potentially lead to erroneous RDCs. 
A tremendous advantage of the presented approach is that couplings with varying alignments can be measured in the same sample using the stretching apparatus. This way errors resulting from separate preparations of isotropic and aligned samples can be excluded.

\section{CONCLUSION}

Using the principle setup provided by Kuchel et al. with gelatin [14], we were able to demonstrate that a rubber-based stretching apparatus can be used to induce tunable alignment for a number of hydrogels and a gel consisting of poly(acrylonitrile) and DMSO. ${ }^{1} \mathrm{~T}_{\mathrm{CH}}$ splittings can be measured for a number of different alignment strengths, allowing RDC-measurements of high precision from the slope of ${ }^{1} \mathrm{~T}_{\mathrm{CH}}$ couplings $v s$ the quadrupolar coupling $\Delta \mathrm{v}_{\mathrm{Q}}$ of the deuterated solvent. The ease of scaling the alignment, the relatively good shimming properties of the apparatus and the full compatibility with standard $5 \mathrm{~mm}$ high resolution NMR equipment make the method widely applicable to the structure determination of all kinds of polar molecules, including e.g. most compounds of pharmaceutical interest and biomacromolecules. The extension of the approach to apolar solvents seems to be possible and is currently under investigation in our laboratory.

\section{ACKNOWLEDGEMENT}

B. Luy thanks the Fonds der Chemischen Industrie and the Deutsche Forschungsgemeinschaft (Heisenberg fellowship LU 835/2-1 and grant LU 835/4-1) and B. Laufer thanks the International Graduate School for Science and Engineering for financial support. We gratefully acknowledge the substantial support and the scientific atmosphere provided by Horst Kessler and his group. We very much thank Robert Fisher for reading the manuscript and we also thank Naumann Gelatine und Leim GmbH (Memmingen, Germany) for providing us generously with high quality gelatin.

\section{SUPPLEMENTARY MATERIAL}

Photos of the several gels within the stretching apparatus and of a PAN/DMSO gel at various stages of extension, the resulting phase slopes with corresponding error analyses for the data presented in Fig. (3), as well as the assignment and a CLIP-HSQC recorded on the hexapeptide are provided. It can be Viewed at www.bentham.org/open/tospecj

\section{REFERENCES}

[1] Saupe A. Kernresonanz in kristallinen Flüssigkeiten und in kristallinflüssigen Lösungen, Teil 1. Z Naturfors 1964; 19a: 161.

[2] Bax A. Weak alignment offers new NMR opportunities to study protein structure and dynamics. Protein Sci 2003; 12: 1-16.

[3] Dong RY. Nuclear Magnetic Resonance of Liquid Crystals. 2nd ed. Springer: New York; 1997.

[4] Samulski ET. Investigations of polymer-chains in oriented fluid phases with deuterium nuclear magnetic-resonance. Polymer 1985; 26: $177-89$.

[5] Tycko R, Blanco FJ, Ishii Y. Alignment of biopolymers in strained gels: A new way to create detectable dipole-dipole couplings in highresolution biomolecular NMR. J Am Chem Soc 2000; 122: 9340-1.
[6] Sass HJ, Musco G, Stahl SJ, Wingfield PT, Grzesiek S. Solution NMR of proteins within polyacrylamide gels: diffusional properties and residual alignment by mechanical stress or embedding of oriented purple membranes. J Biomol NMR 2000; 18: 303-9.

[7] Luy B, Kobzar K, Knör S, Furrer J, Heckmann D, Kessler H. Orientational properties of stretched polystyrene gels in organic solvents and the supression of their residual ${ }^{1} \mathrm{H}$ NMR signals. J Am Chem Soc 2005; 127: 6459-65.

[8] Freudenberger JC, Spitteler P, Bauer R, Kessler H, Luy B. Stretched polydimethylsiloxane gels as NMR-Alignment media for apolar and weakly polar organic solvents: ideal tool for measuring RDCs at low molecular concentrations. J Am Chem Soc 2004; 126: 14690-1.

[9] Freudenberger JC, Knör S, Kobzar K, et al. Stretched polyvinylacetate-gels as NMR-alignment media for the measurement of residual dipolar couplings in polar organic solvents. Angew Chem Int Ed 2005; 44: 423-6.

[10] Haberz P, Farjon J, Griesinger C. A DMSO compatible orienting medium: towards the investigation of the stereochemistry of natural products. Angew Chem Int Ed 2005; 44: 427-9.

[11] Kummerlöwe G, Auernheimer J, Lendlein A, Luy B. Stretched poly(acrylonitrile) as a scalable alignment medium for DMSO. J Am Chem Soc 2007; 129: 6080-1.

[12] Gil RR, Gayathri C, Tsarevsky NV, Matyjaszewski K. Stretched poly(methyl methacrylate) gel aligns small organic molecules in chloroform stereochemical analysis and diastereotopic proton NMR assignment in ludartin using residual dipolar couplings and ${ }^{3} \mathrm{~J}$ coupling constant analysis. J Org Chem 2008; 73: 840-8.

[13] Kobzar K, Kessler H, Luy B. Streched gelatin gels as chiral alignment media for the discrimination of enantiomers by NMR spectroscopy. Angew Chem Int Ed 2005; 44: 3145-7.

[14] Kuchel PW, Chapman BE, Mueller N, Bubb WA, Philp DJ, Torres AM. Apparatus for rapid adjustment of the degree of alignment of NMR samples in aqueous media: Verification with residual quadrupolar splittings in ${ }^{23} \mathrm{Na}$ and ${ }^{133} \mathrm{Cs}$ spectra. J Magn Reson 2006; 180: 256-65.

[15] Naumann C, Bubb WA, Chapman BE, Kuchel PW. Tunablealignment chiral system based on gelatin for NMR spectroscopy. J Am Chem Soc 2007; 129: 5340-1.

[16] Enthart A, Freudenberger JC, Furrer J, Kessler H, Luy B. The CLIP/CLAP-HSQC: Pure absorptive spectra for the measurement of one-bond couplings. J Magn Reson 2008; 192: 314-22.

[17] Kobzar K, Skinner TE, Khaneja N, Glaser SJ, Luy B. Exploring the limits of broadband excitation and inversion pulses. J Magn Reson 2004; 170: 236-43.

[18] Chou JJ, Gaemers S, Howder B, Louis JM, Bax A. A simple apparatus for generating stretched polyacrylamide gels, yielding uniform alignment of proteins and detergent micelles. J Biomol NMR 2001; 21:377-82.

[19] Mangoni A, Esposito V, Randazzo A. Configuration assignment in small organic molecules via residual dipolar couplings. Chem Commun 2003; 154-5.

[20] Furrer J, John M, Kessler H, Luy B. J-spectroscopy in the presence of residual dipolar couplings: determination of one-bond coupling constants and scalable resolution. J Biomol NMR 2007; 37: 231-43.

[21] Luy B, Kobzar K, Kessler H. An easy and scalable method for the partial alignment of organic molecules for measuring residual dipolar couplings. Angew Chem Int Ed 2004; 43: 1092-4.

[22] Courtieu J, Bayle JP, Fung BM. Variable angle sample spinning NMR in liquid crystals. Prog Nucl Magn Reson Spectrosc 1994; 26: 141-69.

[23] Thiele CM. Scaling the alignment of small organic molecules in substituted polyglutamates by variable-angle sample spinning. Angew Chem Int Ed 2005; 44: 2787-90.

[24] Klages J, Neubauer C, Coles M, Kessler H, Luy B. Structure refinement of cyclosporin A in chloroform by using RDCs measured in a Stretched PDMS-gel. Chembiochem 2005; 6: 1672-8. 